\title{
CD69 surface expression on human lung eosinophils after segmental allergen provocation
}

\author{
P. Julius, W. Luttmann, B. Knoechel, C. Kroegel, H. Matthys, J.C. Virchow Jr
}

CD69 surface expression on human lung eosinophils after segmental allergen provocation. P. Julius, W. Luttmann, B. Knoechel, C. Kroegel, H. Matthys, J.C. Virchow Jr. (C)ERS Journals Ltd 1999.

ABSTRACT: CD69 expression on eosinophils is observed in asthma and has been proposed as a marker of essinophil activation. The role of allergens in the in vivo regulation of CD69 expression on eosinophils, however, remains incompletely understood. It was therefore investigated whether CD69 expression on eosinophils can be induced by allergen provocation in vivo.

Ten allergic asthmatics were studied by segmental allergen provocation. Two segments of the right and left lung were challenged with allergen or saline. CD69 expression was determined by flow cytometry and concentrations of interleukins were analysed by enzyme-linked immunosorbent assay in bronchoalveolar lavage (BAL) fluid.

Expression of CD69 on BAL eosinophils in the segments lavaged 10 min following saline instillation (28.3 \pm 8.8 specific mean fluorescence (SMF)) was not significantly different to segments lavaged $10 \mathrm{~min}$ after allergen (80.2 $\pm 21.8 \mathrm{SMF})$ and segments lavaged $18 \mathrm{~h}$ after saline challenge (87.2 $\pm 23.3 \mathrm{SMF})$. However, CD69 expression on eosinophils increased significantly $18 \mathrm{~h}$ after allergen challenge (128.6 $\pm 21.9 \mathrm{SMF}$, $\mathbf{p}<0.03$ ) which was accompanied by elevated granulocyte-macrophage colony-stimulating factor (GM-CSF) concentrations $\left(114.9 \pm 42.9 \mathrm{pg} \cdot \mathrm{mL}^{-1}, \mathrm{p}<0.05\right)$. CD69 expression on eosinophils and GM-CSF concentrations correlated $18 \mathrm{~h}$ following allergen provocation $(r=0.7, p<0.025)$.

These results suggest that in allergic asthma there is an allergen dependent, endobronchial upregulation of eosinophil activation as assessed by CD69 expression on eosinophils.

Eur Respir J 1999; 13: 1253-1259.
Dept of Pneumology, University Medicar Clinic, Freiburg, Germany

Correspondence: W. Luttmann

Dept of Pneumology

University Medical Clinic

Hugstetter Str. 55

79106 Freiburg

Germany

Fax: 497612703704

Keywords: Allergen challenge

CD69

eosinophils

granulocyte-macrophage colony-

stimulating factor

interleukin-4

Received: September 151998

Accepted after revision February 21999

This work was supported by the Forschungskommission, Universitätsklinik Freiburg and by a grant from the Bundesministerium für Bildung, Wissenschaft, Forschung, und Technologie (BMBF 01 GC 9701/7). P. Julius and W. Luttmann contributed equally to this work.
Infiltration of activated eosinophils into the bronchial mucosa is regarded as a specific feature of asthma. Expression of CD69 has been described as a marker of eosinophil activation $[1,2]$. Cross-linking of CD69 with specific monoclonal antibodies can induce eosinophil apoptosis [3], suggesting a role for this surface antigen in the regulation of eosinophilic inflammation. The natural ligand for CD69, however, remains unknown. Increased expression of CD69, a type II integral protein with a Ctype lectin binding domain [4], on eosinophils has been reported in eosinophilic pneumonia and bronchial asthma. Bronchoalveolar lavage (BAL) eosinophils from patients with eosinophilic pneumonia had significantly increased expression of CD69, which was not observed on eosinophils from peripheral blood $[5,6]$. Similarly, in allergic asthma elevated expression of CD69 on eosinophils has been reported in BAL, but not on peripheral blood eosinophils [1]. In contrast, a marked elevation of CD69 expression has been reported on eosinophils from patients with atopic dermatitis only following incubation of eosinophils in vitro [7], while in parasitic infections CD69 expression on peripheral blood eosinophils was significantly increased [8]. The factors regulating CD69 expression on eosinophils in vivo, however, remain unclear. In vitro granulocyte-macrophage colony-stimulating factor (GM-CSF), interleukin (IL)-3, IL-5, IL-13 and phorbol ester have been shown to induce CD69 expression on eosinophils $[1,5,9]$. Incubation of freshly purified eosinophils with GM-CSF led to a very rapid induction of CD69 with expression being detectable after stimulation with GM-CSF for only $1 \mathrm{~h}$ [1]. In atopic dermatitis, CD69 expression on eosinophils in vitro has been associated with an autocrine production of cytokines, possibly GM-CSF or IL-5 [7].

Therefore, in order to elucidate whether CD69 expression on eosinophils can be induced by allergen provocation, which has been associated with increased concentrations of GM-CSF, IL-4 and IL-5 [10-13], CD69 expression on peripheral blood and BAL eosinophils was investigated following segmental allergen provocation in vivo.

\section{Material and methods}

\section{Patients}

Ten allergic asthmatics, eight males and two females, mean age $26.3 \pm 1.52$ yrs with a duration of asthma of $>2$ yrs (mean duration 9.2 $\pm 1.46 \mathrm{yrs}$ ) were studied. All patients suffered from allergic asthma as previously defined [14]. There was a history of intermittent wheeze, chest tightness, cough and sputum production either spontaneously or on allergen provocation, and a bronchial 
hyperreactivity as determined by a modified bronchoprovocation test with carbachol [15]. Each patient had a positive skin prick test to either birch pollen $(n=3)$, rye pollen $(n=5)$, or house dust mite allergen $(n=2)$ extracts (Allergopharma, Reinbek, Germany) and almost all had elevated total immunoglobulin (Ig)E levels (626.8 \pm 249.32 $\left.\mathrm{kU} \cdot \mathrm{L}^{-1}\right)$ as well as specific IgE levels $\left(30.24 \pm 9.53 \mathrm{kU} \cdot \mathrm{L}^{-1}\right)$ (Kabi Pharmacia CAP System, Uppsala, Sweden) to their respective allergen as well as a history of reversible bronchoconstriction after inhalation of these particular allergens. Only one patient had a low total IgE level, but a clear history of allergen induced bronchoconstriction and an elevated specific IgE concentration. There was no history or clinical evidence in any of the patients suggesting a respiratory tract infection prior to or at the time of the segmental allergen challenge. All patients were nonsmokers. Baseline forced expiratory volume in one second (FEV1) was $3.92 \pm 0.27 \mathrm{~L}$ (95.4 $\pm 2.88 \%$ predicted) [16]. All patients received inhaled $\beta_{2}$-agonist therapy on an as needed basis. Cromoglycate $(\mathrm{n}=3)$ and inhaled corticosteroids $(n=2)$ were withheld 7 days prior to entry into the study. All patients gave their written informed consent. The study protocol was approved by the Ethics Committee of the University of Freiburg.

\section{Inhaled allergen provocation}

Prior to the segmental allergen challenge, eight patients underwent an inhaled allergen challenge as previously described [17] to establish dual bronchial reactions to the inhaled allergen and to determine the individual provocative dose causing a $20 \%$ fall in FEV1 (PD20) for the respective allergen (two patients were challenged with a dose of 50 protein nitrogen unit (PNU)).

\section{Segmental allergen challenge}

Bronchoscopy was performed as previously described [17] using an Olympus BF 1T30 (Hamburg, Germany) via the oral or nasal route following local anaesthesia with Novesine §) (Wander, Bern, Switzerland).

\section{Determination of $I L-4, I L-5$, and GM-CSF}

IL-4 and IL-5 were assessed in BAL fluid as previously described [17]. GM-CSF in BAL fluid was assessed using monoclonal anti-human cytokine antibodies (Clone D223B6; PharMingen, San Diego, CA, USA) as the capture antibody and biotin-conjugated monoclonal anti-human cytokine antibodies (Clone BVD2-21C11; PharMingen) in a concentration of $2 \mu \mathrm{g} \cdot \mathrm{mL}^{-1}$ as previously described [9]. The results were obtained from a standard curve established with human recombinant GM-CSF (Pharma Biotechnologie Hannover (PBH), Hannover, Germany). Measurements were performed in duplicate and are expressed as means from both determinations. The sensitivity of the cytokine assays was below $3 \mathrm{pg} \cdot \mathrm{mL}^{-1}$ for each of the cytokines measured.

\section{Flow cytometric analysis}

Peripheral blood and BAL samples were processed and cells were counted by flow cytometry as described in detail previously [17]. After lysis of erythrocytes, $20 \mu \mathrm{L}$ of either whole blood or cells from BAL were incubated in the presence of saturating concentrations of phycoerythrin (PE)-conjugated CD69 (Becton Dickinson, San Jose, CA, USA) and fluorescein isothiocyanate (FITC)-conjugated CD16 (Immunotech, Marseille, France) or PEconjugated anti-IgG (DAKO, Hamburg, Germany) and FITC-conjugated CD16, respectively, in the dark on ice for $30 \mathrm{~min}$. The cells were washed twice with phosphatebuffered saline (PBS; Dulbecco, Berlin, Germany) containing 2\% foetal calf serum (FCS) and subjected to cytofluorometric analysis which was performed on $1 \times$ $10^{4}$ cells from each sample by using laser excitation at $585 \mathrm{~nm}$ (PE) and $503 \mathrm{~nm}$ (FITC), respectively. Nonspecific fluorescence was detected as previously described [17] and subtracted from the mean fluorescence measured with anti-CD69 antibodies. CD16 fluorescence was used to separate CD16 negative and CD49d (Becton Dickinson) positive eosinophils from CD16 positive neutrophils (fig. 1). In initial experiments it could be demonstrated that within the cell population gated accordingly only very few were CD14 positive.

\section{Purification of eosinophils}

Eosinophils were obtained from $100 \mathrm{~mL}$ ethylenediamine tetraacetic acid (EDTA)-blood of healthy donors. Cells were separated by negative immunomagnetic selection as previously described [9]. Comparing CD14+ and CD16- cells according to granularity (side scatter) and size (forward scatter) it could be shown that the cells gated according to CD16- and CD49+ contained only very few CD14 positive cells.

\section{Cell culture}

Purified eosinophils $\left(1 \times 10^{6}\right.$ cells $\left.\cdot \mathrm{mL}^{-1}\right)$ were cultured as previously described [9] in culture medium alone or in the presence of either GM-CSF or IL-4 (PBH). Before immunofluorescence labelling with anti-CD69 antibodies, the cells were washed twice in PBS containing $2 \%$ FCS.

In vitro stimulation of eosinophils with GM-CSF, tumour necrosis factor- $\alpha$, and histamine

Purified eosinophils from peripheral blood of normal donors were incubated in the presence of GM-CSF (Bioconcept, Umkirch, Germany) (1 and $\left.10 \mathrm{ng} \cdot \mathrm{mL}^{-1}\right)$, tumour necrosis factor (TNF)- $\alpha$ (Bioconcept) $\left(10 \mathrm{ng} \cdot \mathrm{mL}^{-1}\right)$, and histamine (Sigma, Deisenhofen, Germany) $\left(10^{-6}-10^{-4} \mathrm{M}\right)$ for $10 \mathrm{~min}$ and then analysed for CD69 expression. In addition, purified eosinophils from normal donors which had been preincubated ("primed") for $18 \mathrm{~h}$ with a mixture of IL-3, IL-5 (both from Bioconcept) and GM-CSF (0.01 $\mathrm{ng} \cdot \mathrm{mL}^{-1}$ each), which by itself did not induce CD69 expression, were incubated with GM-CSF $\left(1\right.$ and $\left.10 \mathrm{ng} \cdot \mathrm{mL}^{-1}\right)$, TNF- $\alpha\left(10 \mathrm{ng} \cdot \mathrm{mL}^{-1}\right)$, and histamine $\left(10^{-6}-10^{-4} \mathrm{M}\right)$ for 10 min and then analysed for CD69 expression.

\section{Statistical analysis}

Results are expressed as arithmetic mean \pm SEM. Differences between groups were analysed using the Wilcoxon matched pairs test. Differences with p-values $<0.05$ were considered statistically significant. Relationships are expressed using Pearson's rank correlation. 
a)

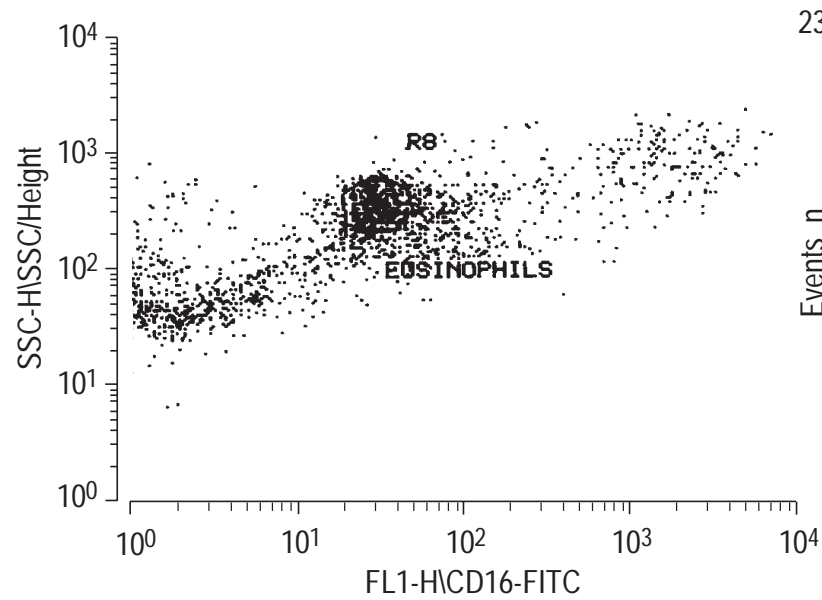

c)

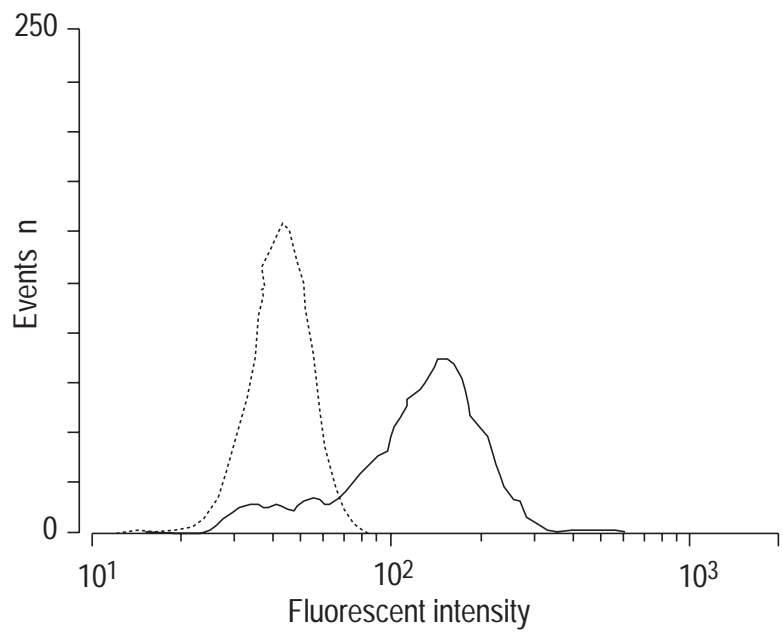

b)

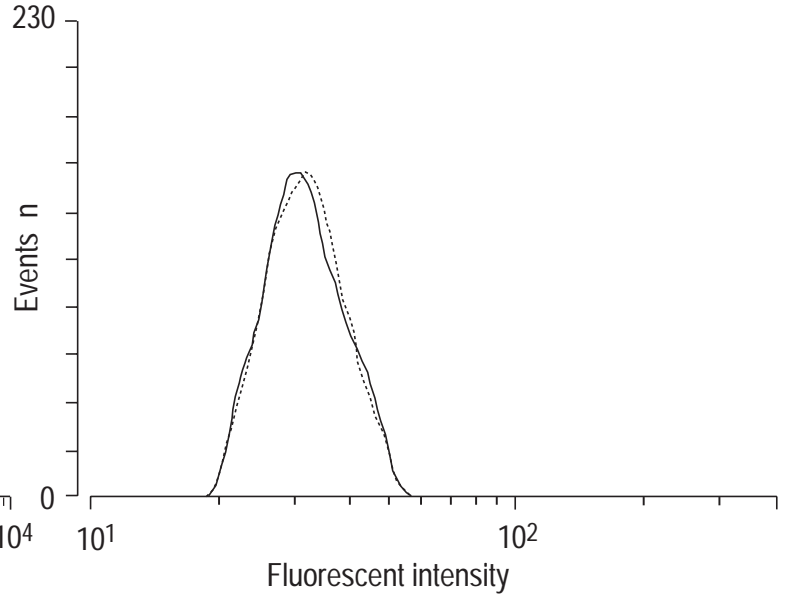

d)

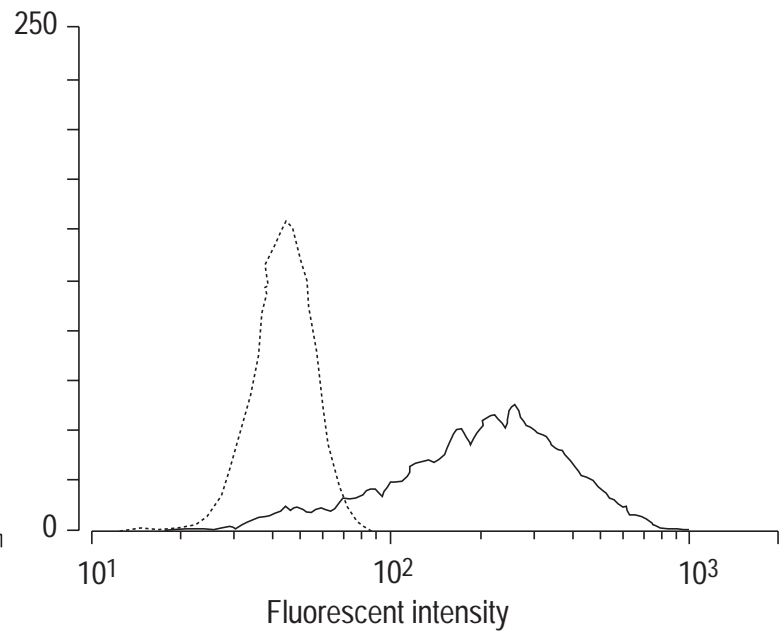

Fig. 1. - CD16, CD49d and CD69 expression on bronchoalveolar lavage (BAL) eosinophils $18 \mathrm{~h}$ after allergen provocation. Cells were obtained from BAL $18 \mathrm{~h}$ after allergen provocation and incubated with fluorescent anti-CD16 (fluorescein isothiocyanate (FITC)), anti-CD49d (phycoerythin (PE)) and anti-CD69 antibodies. The dot plot (a) shows ungated BAL cells. Eosinophils (R8) were gated and their CD16 (b), CD49d (c) and CD69 (d) expressions $(-)$ are shown in comparison to an unspecific control antibody (Immunoglobulin $(\mathrm{Ig}) \mathrm{G} ; \cdots \cdots)$. SSC: side scatter.

\section{Results}

Eosinophils in peripheral blood prior to and after segmental allergen provocation

There was a significant increase in the total cell number in peripheral blood $18 \mathrm{~h}$ after segmental allergen provocation $\left(8.5 \pm 0.6 \times 10^{3}\right.$ cells $\left.\mu \mathrm{L}^{-1}\right)$ compared to baseline $\left(5.9 \pm 0.4 \times 10^{3}\right.$ cells $\left.\mu \mathrm{L}^{-1} ; \mathrm{p}<0.01\right)$. Among the different cell populations the total number of eosinophils showed a slight, but not significant, increase $\left(0.2 \pm 0.1 \times 10^{3}\right.$ cells $\cdot \mu \mathrm{L}^{-1}$ before allergen challenge and $0.3 \pm 0.1 \times 10^{3}$ cells $\mu \mathrm{L}^{-1} 18 \mathrm{~h}$ after allergen challenge), while the relative number of eosinophils did not change $(4.2 \pm 0.7 \%$ before and $3.9 \pm 0.7 \%$ $18 \mathrm{~h}$ after allergen challenge).

Eosinophils in BAL following segmental allergen provocation

Following allergen provocation a marked increase in eosinophils was observed in the allergen challenged seg- ment after $18 \mathrm{~h}$ which was significantly elevated compared to the other segments lavaged 10 min after allergen challenge or $10 \mathrm{~min}$ and $18 \mathrm{~h}$ after saline challenge (table 1). This increase in eosinophils, which was consistently observed in all patients, was associated with a statistically significant increase in the relative number of eosinophils $18 \mathrm{~h}$ after segmental allergen challenge compared to the other segments $(1.5 \pm 0.7 \%$ and $3.8 \pm 1.5 \% 10 \mathrm{~min}$ and $18 \mathrm{~h}$ after $\mathrm{NaCl}$ instillation, and $1.7 \pm 0.5 \%$ and $28.7 \pm 6.1 \% 10$ min and $18 \mathrm{~h}$ after allergen challenge; $\mathrm{p}<0.03$ ).

CD69 expression on eosinophils and neutrophils following segmental allergen provocation

CD69 expression on peripheral blood eosinophils was $16.2 \pm 3.2$ specific mean fluorescence (SMF) before allergen challenge and increased only slightly $18 \mathrm{~h}$ after allergen provocation (22.5 $\pm 6.3 \mathrm{SMF})$. However, this difference failed to reach statistical significance. In contrast to peripheral blood eosinophils there was a marked upregulation in the expression of CD69 on eosinophils obtained from BAL fluid following segmental allergen provocation (fig. 2). 
Table 1. - Cellular composition in bronchoalveolar lavage fluid after segmental allergen provocation $\left(\right.$ cells $\times 10^{3} \cdot \mathrm{mL}^{-1}$; mean \pm SEM)

\begin{tabular}{lrccc}
\hline & Macrophages & Neutrophils & Eosinophils & Lymphocytes \\
\hline C 10 min & $75.4 \pm 28.2$ & $0.5 \pm 0.3$ & $3.1 \pm 1.9$ & $27.2 \pm 9.5$ \\
C 18 h & $190.2 \pm 52.9$ & $13.4 \pm 4.5$ & $6.8 \pm 2.3$ & $31.3 \pm 5.5$ \\
P 10 min & $61.4 \pm 13.1$ & $0.7 \pm 0.3$ & $1.6 \pm 0.6$ & $17.2 \pm 4.8$ \\
P 18 h & $129.2 \pm 29.2$ & $41.7 \pm 11.9$ & $125.0 \pm 44.7$ & $77.1 \pm 15.0$
\end{tabular}

C 10 min: saline challenged control segment lavaged $10 \mathrm{~min}$ after instillation of $2.5 \mathrm{~mL}$ normal saline; $\mathrm{C} 18 \mathrm{~h}$ : saline challenged control segment lavaged $18 \mathrm{~h}$ after instillation of $2.5 \mathrm{~mL}$ normal saline; P $10 \mathrm{~min}$ : allergen challenged segment lavaged 10 min after instillation of allergen $10 \times$ provocative dose causing a $20 \%$ fall in forced expiratory volume in one second (PD20). P $18 \mathrm{~h}$ : allergen challenged segment lavaged $18 \mathrm{~h}$ after instillation of allergen $10 \times$ PD20. Cell numbers are given in absolute numbers.

BAL eosinophils showed a significant $(\mathrm{p}<0.03)$ increase

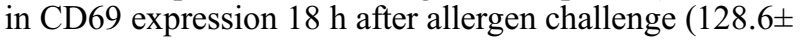
22.0 SMF) compared to $10 \mathrm{~min}$ after allergen challenge $(80.2 \pm 21.8 \mathrm{SMF})$ as well as $10 \mathrm{~min}(28.3 \pm 8.8 \mathrm{SMF})$ and $18 \mathrm{~h}$ after $\mathrm{NaCl}$ instillation (87.2 $\pm 23.3 \mathrm{SMF}$ ) (fig. 2a).

Similar to peripheral blood eosinophils, there was no significant change in CD69 expression on peripheral blood neutrophils before and $18 \mathrm{~h}$ after segmental allergen challenge (22.0 $\pm 10.6 \mathrm{SMF}$ and 14.1 $\pm 6.4 \mathrm{SMF}$, respectively; NS). In contrast to BAL eosinophils there was also no significant change in CD69 expression on BAL neutrophils (10 min after $\mathrm{NaCl}$ instillation $14.9 \pm 6.4 \mathrm{SMF}$; $18 \mathrm{~h}$ after $\mathrm{NaCl}$ instillation $43.0 \pm 20.8 \mathrm{SMF} ; 10 \mathrm{~min}$ after allergen challenge $25.6 \pm 14.5 \mathrm{SMF} ; 18 \mathrm{~h}$ after allergen challenge

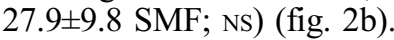

\section{Cytokines in BAL fluid}

The T-helper (Th)2-cytokines IL-4, IL-5 and GM-CSF measured in unconcentrated BAL fluid were all significantly elevated $18 \mathrm{~h}$ after allergen challenge (table 2). IL-4 was markedly increased compared to the segments lavaged 10 min after allergen challenge and $18 \mathrm{~h}$ after saline instillation. Similarly, there was a significant increase in the IL-5 concentrations measured $18 \mathrm{~h}$ after segmental allergen provocation compared to the segment lavaged 10 min following allergen challenge and $18 \mathrm{~h}$ after saline instillation. Finally, significantly elevated concentrations of GM-CSF were measured $18 \mathrm{~h}$ following allergen provocation (table 2 ).

Correlation between cytokine concentrations in $B A L$ and CD69 expression on eosinophils

Among the different cytokines measured in BAL fluid a weak, although statistically significant, correlation was observed between CD69 expression on bronchoalveolar eosinophils and the GM-CSF concentrations measured in BAL fluid $18 \mathrm{~h}$ after segmental allergen provocation ( $\mathrm{r}=$ $0.697, \mathrm{p}<0.025$ ) (fig. 3a). Furthermore, there was a trend towards a correlation of CD69 expression on eosinophils and IL-4 concentrations in BAL fluid. However, this relationship failed to reach statistical significance $(\mathrm{r}=0.58$, p<0.077) (fig. 3b).

\section{Effect of GM-CSF and IL-4 on CD69 expression on isolated eosinophils}

In order to investigate the significance of the observed correlation between eosinophil CD69 expression and GM-
CSF or IL-4 concentrations in BAL fluid, freshly purified eosinophils from healthy volunteers were incubated with two different concentrations of GM-CSF and IL-4 (1 and $10 \mathrm{ng} \cdot \mathrm{mL}^{-1}$ ) in vitro for $6 \mathrm{~h}$. CD69 expression was then measured by flow cytometry. Only a weak expression of CD69 was observed when freshly isolated eosinophils from normal subjects were incubated in medium alone for $6 \mathrm{~h}$. However, incubation with either 1 or $10 \mathrm{ng} \cdot \mathrm{mL}^{-1}$ of GM-CSF resulted in a marked increase in CD69 expression as shown in figure $4 \mathrm{a}$, which was also observed following incubation with IL-4, although to a lesser extent. In addition, when the effects of GM-CSF and IL-4 (both $10 \mathrm{ng} \cdot \mathrm{mL}^{-1}$ ) on the induction of CD69 expression on eosinophils were measured over time, a similar increase was observed between 2 and $6 \mathrm{~h}$. This increased expression of CD69 on eosinophils persisted for $24 \mathrm{~h}$ after incubation with GM-CSF, but showed a small decline with IL-4 (fig. 4b).

In vitro stimulation of eosinophils with GM-CSF, TNF$\alpha$, and histamine

In order to analyse which factors might be involved in the rapid upregulation of CD69 on BAL eosinophils recovered $10 \mathrm{~min}$ after allergen challenge, purified cells from peripheral blood of normal donors were incubated for $10 \mathrm{~min}$ in the presence of GM-CSF, TNF- $\alpha$, and histamine which can be released from mast cells immediately following allergen challenge. As shown in table 3, none of these mediators influenced CD69 expression on peripheral blood eosinophils from normal donors.

\section{Discussion}

Infiltration of activated eosinophils into the bronchial lumen is a prominent feature of asthma [18] and has been associated with specific, eosinophil-mediated damage to the respiratory epithelium. CD69 which is expressed on a number of cells has been described as a marker for eosinophil activation $[1,2]$. Several studies have reported elevated expression of this surface antigen on BAL eosinophils compared to peripheral blood eosinophils in diseases such as eosinophil pneumonia and asthma $[1,5$, 6]. While CD69 expression on eosinophils in vitro can be induced by incubation with cytokines such as IL-3, IL-5, and GM-CSF [1], the factors leading to CD69 expression on eosinophils in vivo remain unclear. Therefore, in this study, it was investigated whether allergen exposure can modify CD69 expression and thus indicate eosinophil activation in vivo. 

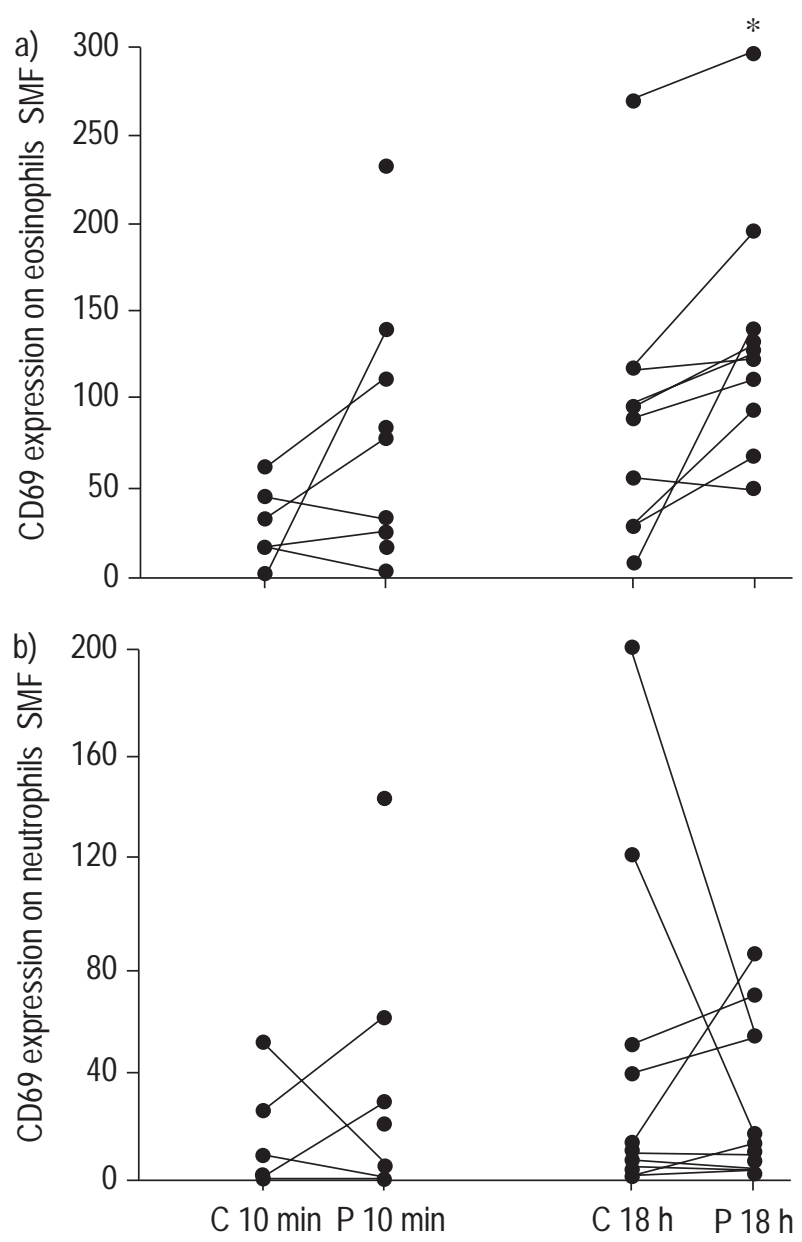

Fig. 2. - CD69 expression on bronchoalveolar lavage (BAL) eosinophils (a) and neutrophils (b). Bronchoalveolar cells were obtained from mild asthmatics after saline $(\mathrm{C})$ or allergen provocation $(\mathrm{P})$ at $10 \mathrm{~min}$ and $18 \mathrm{~h}$ after instillation. Eosinophils and neutrophils were analysed by flow cytometry after labelling with CD69 specific antibody. Data are presented as specific mean fluorescence $(\mathrm{SMF}) \pm$ SEM of 10 different patients. ${ }^{*}$ : p<0.05, compared to $\mathrm{C} 10 \mathrm{~min}, \mathrm{P} 10 \mathrm{~min}$ and $\mathrm{C} 18 \mathrm{~h}$. For definitions of treatments see footnote to table 1 .

Using the model of segmental allergen provocation this is the first study to show that CD69 expression is indeed markedly upregulated on BAL eosinophils following allergen provocation. The study thus extends the observations of HARTNELL et al. [1] by showing that the low basal expression of CD69 on BAL eosinophils increases following allergen exposure and therefore provides further evidence for the hypothesis that eosinophil activation in allergic asthma in vivo is a dynamic process which is allergen dependent. In contrast to eosinophils, BAL neutrophils showed a baseline expression of CD69 which did not change significantly following allergen provocation. Therefore, from the present study it was concluded that CD69 expression in this model of allergic asthma appears to be specific for eosinophils.

Interestingly, a small increase in CD69 expression on eosinophils was also observed in the segment lavaged 10 min after allergen provocation, at a timepoint where infiltration of newly activated eosinophils is unlikely. Although this difference in CD69 expression between the two segments lavaged after $10 \mathrm{~min}$ following either saline or
Table 2. - Cytokine concentrations in bronchoalveolar lavage fluid after segmental allergen provocation $\left(\mathrm{pg} \cdot \mathrm{mL}^{-1}\right.$; mean \pm SEM)

\begin{tabular}{lccc}
\hline & IL-4 & IL-5 & GM-CSF \\
\hline C 10 min & $8.2 \pm 6.2$ & $9.7 \pm 5.7$ & $7.9 \pm 4.4$ \\
C 18 h & $11.5 \pm 9.2$ & $4.8 \pm 4.3$ & $8.5 \pm 3.6$ \\
P 10 min & $5.6 \pm 3.5$ & $8.3 \pm 5.1$ & $8.2 \pm 3.3$ \\
P 18 h & $131.6 \pm 56.1^{*}$ & $198.0 \pm 67.2^{*}$ & $114.9 \pm 42.9^{*}$ \\
\hline
\end{tabular}

Cytokine concentrations of interleukin (IL)-4, IL-5 and granulocyte-macrophage colony-stimulating factor (GM-CSF) in BAL fluid in the segments lavaged $10 \mathrm{~min}$ and $18 \mathrm{~h}$ after saline instillation (C $10 \mathrm{~min}$ and $\mathrm{C} 18 \mathrm{~h}$ ) as well as the segments lavaged 10 min and $18 \mathrm{~h}$ after allergen provocation $(\mathrm{P} 10 \mathrm{~min}$ and $\mathrm{P} 18 \mathrm{~h}$ ). *: $\mathrm{p}<0.05$ for $\mathrm{P} 18 \mathrm{~h}$ compared to P $10 \mathrm{~min}$, and C $18 \mathrm{~h}$ for IL-4 and for $\mathrm{P} 18 \mathrm{~h}$ compared to all other segments for IL-5 and for GM-CSF.

allergen challenge was not statistically significant these findings would support the concept that CD69 expression on eosinophils can be upregulated rapidly, possibly due to exposure of intracellularly stored receptors [9]. In order to elucidate factors which might regulate this rapid increase in CD69 expression, peripheral blood eosinophils from normal donors were incubated with GM-CSF, TNF- $\alpha$, and histamine. These factors which are rapidly released following allergen-dependent mast cell activation, however, did not change CD69 expression on eosinophils in
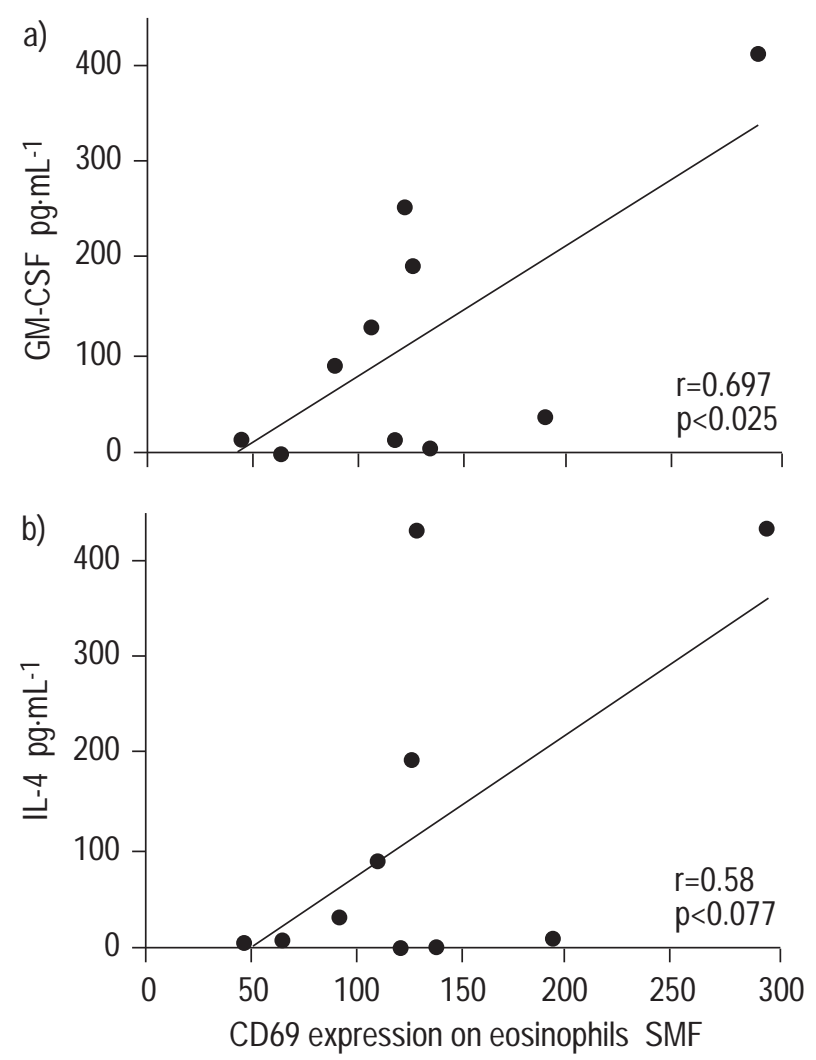

Fig. 3. - Correlation between CD69 expression on eosinophils and cytokine concentration in bronchoalveolar lavage (BAL). Eosinophil CD69 expression $18 \mathrm{~h}$ after segmental allergen provocation correlated with a) granulocyte-macrophage colony-stimulating factor (GM-CSF) concentrations $(\mathrm{r}=0.697, \mathrm{p}<0.025)$ and $\mathrm{b})$ interleukin (IL)-4 concentrations $(\mathrm{r}=0.58, \mathrm{p}<0.077)$ in BAL $18 \mathrm{~h}$ after segmental allergen provocation. SMF: specific mean fluorescence. 

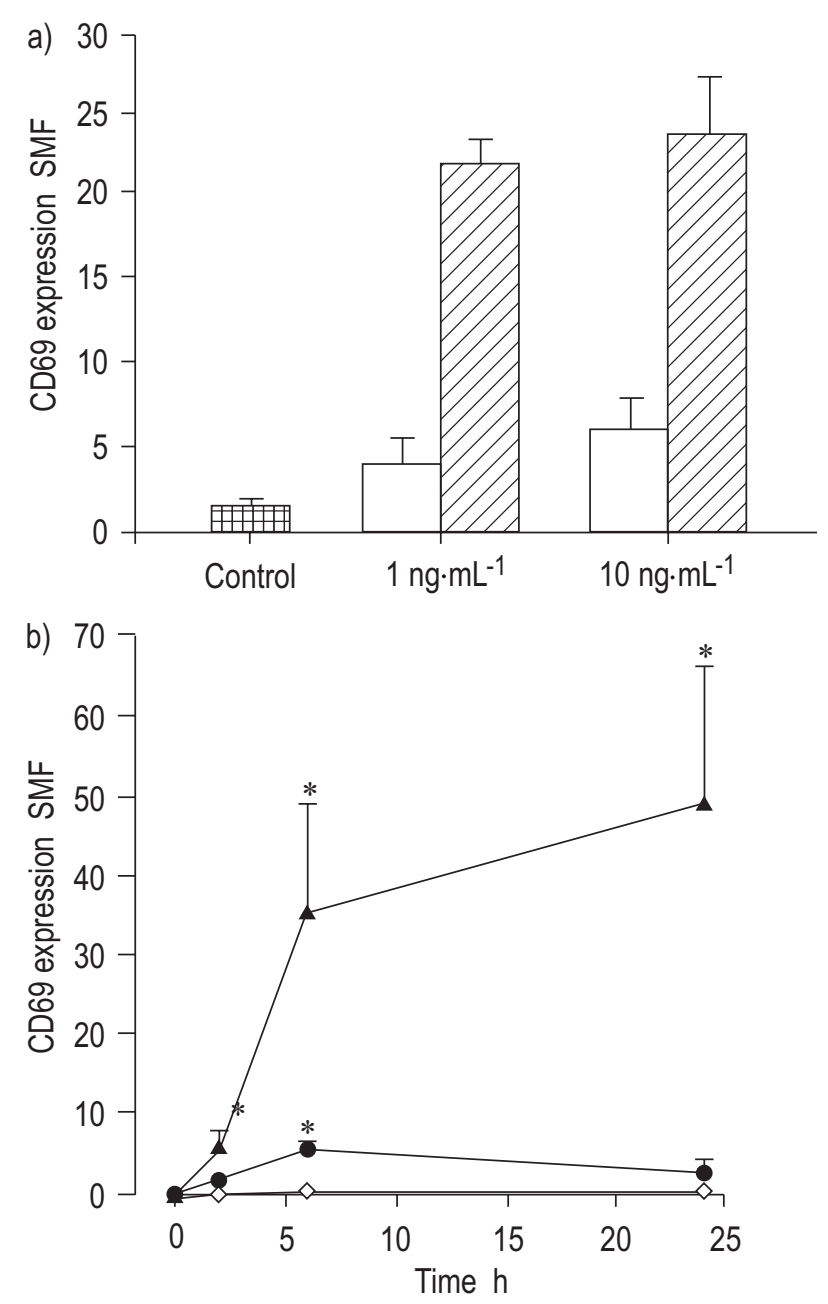

Fig. 4. - CD69 expression on isolated human peripheral blood eosinophils after stimulation with interleukin (IL)-4 and granulocytemacrophage colony-stimulating factor (GM-CSF). a) Isolated peripheral blood eosinophils were stimulated with 1 and $10 \mathrm{ng} \cdot \mathrm{mL}^{-1}$ of either IL-4 $(\square)$ or GM-CSF $(\mathbb{Z})$. Cells cultured in medium alone served as controls (曲). CD69 expression was measured after $6 \mathrm{~h}$ in culture. b) Isolated peripheral blood eosinophils were stimulated with $10 \mathrm{ng} \cdot \mathrm{mL}^{-1}$ of either IL-4 $(\bullet)$ or GM-CSF $(\boldsymbol{\Delta})$ for 2, 6 and $24 \mathrm{~h}$. Cells cultured in medium alone served as controls $(\diamond)$. Data are given as specific mean fluorescence $(\mathrm{SMF}) \pm \mathrm{SEM}$. $*: \mathrm{p}<0.05$, compared to control cells incubated in medium alone.

vitro. Therefore, it could be speculated that mucosal eosinophils with elevated CD69 expression are preferentially detected in BAL fluid following allergen provocation. Alternatively, unlike eosinophils from normal donors, eosinophils from asthmatic patients might have been exposed to a variety of "priming" factors in vivo that could facilitate a rapid upregulation of CD69 in vivo. However, even the incubation of peripheral blood eosinophils from normal donors with a mixture of IL-3, IL-5, and GM-CSF (which did not cause CD69 upregulation) failed to reproduce the rapid upregulation of CD69 similar to that observed $10 \mathrm{~min}$ following allergen provocation. Thus, the mechanisms responsible for CD69 upregulation in vivo remain to be elucidated. The large, statistically significant increase in CD69 expression $18 \mathrm{~h}$ after segmental allergen challenge which coincided with a large increase in the numbers of eosinophils present in the
Table 3. - CD69 expression following incubation with different concentrations of granulocyte-macrophage colony-stimulating factor (GM-CSF), tumour necrosis factor (TNF)- $\alpha$, histamine and a mixture of interleukin (IL)-3, IL-5 and GM-CSF (mean \pm SEM)

\begin{tabular}{lcr}
\hline & $\begin{array}{c}\text { Priming with } \\
\text { GM-CSF/IL-3/IL-5 }\end{array}$ & No priming \\
\hline Control & $5.35 \pm 4.98$ & $2.24 \pm 0.60$ \\
GM-CSF ng $\cdot \mathrm{mL}^{-1}$ & $3.36 \pm 1.39$ & $1.04 \pm 0.55$ \\
10 & $5.0 \pm 1.71$ & $1.86 \pm 0.85$ \\
TNF- $\alpha 10 \mathrm{ng} \cdot \mathrm{mL}^{-1}$ & & \\
Histamine M & $2.04 \pm 0.65$ & \\
$10^{-6}$ & $1.23 \pm 0.52$ & $-0.21 \pm 0.46$ \\
$10^{-5}$ & $0.1 \pm 0.14$ & $4.25 \pm 2.22$ \\
$10^{-4}$ & \\
\hline
\end{tabular}

CD69 expression following incubation with different concentrations of GM-CSF, TNF- $\alpha$ and histamine for 10 min after "priming" with a mixture of IL-3, IL-5 and GM-CSF.

BAL fluid suggests a selective, allergen-dependent infiltration of activated eosinophils into the site of allergen challenge. Alternatively, it might be possible that eosinophils which are attracted to the site of allergic inflammation are activated by local factors present in the inflamed microenvironment $18 \mathrm{~h}$ following allergen provocation.

In this study, anti-inflammatory therapy was withheld for 7 days prior to segmental allergen challenge. Although an even longer period might have been desirable to exclude any effects of inhaled corticosteroids or cromoglycate on CD69 expression it was considered to be unethical to withhold therapy for longer periods of time, although this was well tolerated by all subjects. However, with respect to this study no differences were observed between patients treated with corticosteroids, cromoglycate or $\beta_{2}$-agonists suggesting that the influence of corticosteroids or cromoglycate on eosinophil CD69 expression did not influence the findings when they were withheld for at least 7 days.

In this study, the increase in CD69 expression on eosinophils was accompanied by an increase in GM-CSF concentration in the allergen challenged segment $18 \mathrm{~h}$ following allergen challenge. A similar increase, which correlated with the number of infiltrating eosinophils in BAL $18 \mathrm{~h}$ after allergen challenge [11], has been reported following allergen provocation [11-13]. Furthermore, several studies have reported an increase in CD69 expression on eosinophils following incubation with GMCSF in vitro $[1,19]$. In the present study this data was confirmed $[1,19]$ by demonstrating that CD69 expression on eosinophils can be induced by incubation of isolated cells with GM-CSF. This does suggest that the increase in CD69 expression and GM-CSF levels observed $18 \mathrm{~h}$ following allergen provocation in this study might be causally related, but supports the assumption that this cytokine plays an important role in the regulation of eosinophil activation in vivo.

Several cells such as mast cells, macrophages and Tlymphocytes have been implicated in the production of GM-CSF [20, 21]. However, in view of previous studies which have shown that eosinophils themselves can produce GM-CSF [22-24] and that the ability of cultured eosinophils to induce CD69 expression by autocrine mechanisms can be inhibited by anti-GM-CSF antibodies 
[7], eosinophils cannot be excluded as a major source of GM-CSF in vivo which might cause autocrine modulation of CD69 expression.

Since there was also a weak, statistically nonsignificant correlation between IL-4 concentrations and CD69 expression on BAL eosinophils $18 \mathrm{~h}$ following allergen provocation it was investigated whether IL-4 can induce CD69 expression on cultured eosinophils in vitro. Interestingly, a small, concentration dependent effect was demonstrated for IL-4 on CD69 expression in this setting, suggesting that in addition to GM-CSF other cytokines with a Th2 phenotype can enhance eosinophil activation. Thus, in view of these in vitro results and the observed relationship between IL-4 and CD69 expression in vivo the hypothesis that IL-4 might also contribute to eosinophil activation cannot be refuted as assessed by CD69 expression following allergen provocation.

In this study, the expression of CD69 on eosinophils in BAL fluid $10 \mathrm{~min}$ following saline instillation was not significantly different to the expression observed on peripheral blood eosinophils. This suggests that, in the absence of allergen eosinophil activation (assessed by CD69 expression) CD69 expression does not differ between BAL and peripheral blood in mild, asymptomatic asthma.

In conclusion, this is the first study to provide evidence that in patients with allergic asthma segmental allergen provocation causes an allergen dependent upregulation of CD69 expression on eosinophils which might, in part, be regulated by locally released cytokines such as granulocytemacrophage colony-stimulating factor and interleukin-4.

Acknowledgements. The authors wish to thank S. Bock and S. Schmidt for their expert technical assistance.

\section{References}

1. Hartnell A, Robinson DS, Kay AB, Wardlaw AJ. CD69 is expressed by human eosinophils activated in vivo in asthma and in vitro by cytokines. Immunology 1993; 80: 281-286.

2. Matsumoto K, Appiah-Pippim J, Schleimer RP, Bickel CA, Beck LA, Bochner BS. CD44 and CD69 represent different types of cell-surface activation markers for human eosinophils. Am J Respir Cell Mol Biol 1998; 18: 860-866.

3. Walsh GM, Williamson ML, Symon FA, Willars GB, Wardlaw AJ. Ligation of CD69 induces apoptosis and cell death in human eosinophils cultured with granulocytemacrophage colony-stimulating factor. Blood 1996; 87: 2815-2821.

4. Testi R, D'Ambrosio D, De Maria R, Santoni A. The CD69 receptor: a multipurpose cell-surface trigger for hematopoietic cells. Immunol Today 1994; 15: 479-483.

5. Nishikawa K, Morii T, Ako B, Hamada K, Saito S, Narita $\mathrm{N}$. In vivo expression of CD69 on lung eosinophils in eosinophilic pneumonia: CD69 as a possible activation marker for eosinophils. J Allergy Clin Immunol 1992; 90: 169-174.

6. Okubo Y, Hossain M, Kai R, et al. Adhesion molecules on eosinophils in acute eosinophilic pneumonia. Am J Respir Crit Care Med 1995; 151: 1259-1262.

7. Wedi B, Raap U, Lewrick H, Kapp A. Delayed eosinophil programmed cell death in vitro: a common feature of inhalant allergy and extrinsic and intrinsic atopic dermatitis. J Allergy Clin Immunol 1997; 100: 536-543.
8. Mawhorter SD, Stephany DA, Ottesen EA, Nutman TB. Identification of surface molecules associated with physiologic activation of eosinophils. Application of wholeblood flow cytometry to eosinophils. J Immunol 1996; 156: 4851-4858.

9. Luttmann W, Knoechel B, Foerster M, Matthys H, Virchow JC Jr, Kroegel C. Activation of human eosinophils by IL-13. Induction of CD69 surface antigen, its relationship to messenger RNA expression, and promotion of cellular viability. J Immunol 1996; 157: 1678-1683.

10. Kroegel C, Julius P, Matthys H, Virchow JC, Luttmann W. Endobronchial secretion of interleukin-13 following local allergen challenge in atopic asthma-relationship to interleukin-4 and eosinophil counts. Eur Respir J 1996; 9: 899-904.

11. Virchow JC Jr, Walker C, Hafner D, et al. T cells and cytokines in bronchoalveolar lavage fluid after segmental allergen provocation in atopic asthma. Am J Respir Crit Care Med 1995; 151: 960-968.

12. Broide DH, Firestein GS. Endobronchial allergen in asthma. Demonstration of cellular source of granulocyte macrophage stimulating factor by in situ hybridisation. $J$ Clin Invest 1991; 88: 1048-1053.

13. Kato M, Iui MC, Stealey BA, et al. Production of granulocyte/macrophage colony-stimulating factor in human airways during allergen-induced late-phase reactions in atopic subjects. Lymphokine Cytokine Res 1992; 11:287-292.

14. Anonymous. International consensus report on diagnosis and treatment of asthma. National Heart, Lung, and Blood Institute, National Institutes of Health. Bethesda, Maryland 20892. Publication no. 92-3091, March 1992. Eur Respir J 1992; 5: 601-646.

15. Cockcroft DW, Killian DN, Mellon JJ, Hargreave FE. Bronchial reactivity to inhaled histamine: a method and clinical survey. Clin Allergy 1977; 7: 235-243.

16. Ruhle KH, Matthys H. Kritische Auswahl von Sollwerten fuer ein Computer-Programm zur Routine-Lungenfunktions-Diagnostik. Pneumonologie 1976; 153: 223-233.

17. Virchow JC Jr, Julius P, Matthys H, Kroegel C, Luttmann W. CD14 expression and soluble CD14 after segmental allergen provocation in atopic asthma. Eur Respir J 1998; 11: 317-323.

18. Bousquet J, Chanez P, Lacoste JY, et al. Eosinophilic inflammation in asthma. N Engl JMed 1990; 323: 1033-1039.

19. Ziegler SF, Ramsdell F, Alderson MR. The activation antigen CD69. Stem Cells 1994; 12: 456-465.

20. Baldwin GC. The biology of granulocyte-macrophage colony-stimulating factor: effects on hematopoietic and nonhematopoietic cells. Dev Biol 1992; 151: 352-367.

21. Robinson DS, Hamid Q, Ying S, et al. Predominant TH-2 like bronchoalveolar T-lymphocyte population in atopic asthma. N Engl J Med 1992; 326: 298-304.

22. Kita H, Ohnishi T, Okubo Y, Weiler D, Abrams JS, Gleich GJ. Granulocyte/macrophage colony-stimulating factor and interleukin 3 release from human peripheral blood eosinophils and neutrophils. $J$ Exp Med 1991; 174: 745748.

23. Alan R, Forsythe P, Stafford S, Fukuda Y. Transforming growth factor beta abrogates the effects of hematopoietins on eosinophils and induces their apoptosis. J Exp Med 1994; 179: 1041-1045.

24. Walsh GM, Wardlaw AJ. Dexamethasone inhibits prolonged survival and autocrine granulocyte-macrophage colony-stimulating factor production by human eosinophils cultured on laminin or tissue fibronectin. $J$ Allergy Clin Immunol 1997; 100: 208-215. 